\title{
Article \\ Semi-Automated High-Throughput Substrate Screening Assay for Nucleoside Kinases
}

\author{
Katja F. Hellendahl ${ }^{1,+}{ }^{\text {, Maryke Fehlau }}{ }^{1,2,+}$, Sebastian Hans ${ }^{1}{ }^{\mathbb{D}}$, Peter Neubauer ${ }^{1}\left(\mathbb{D}\right.$ and Anke Kurreck ${ }^{1,2, *}$ \\ 1 Chair of Bioprocess Engineering, Faculty III Process Sciences, Institute of Biotechnology, \\ Technische Universität Berlin, Ackerstraße 76, 13355 Berlin, Germany; k.hellendahl@tu-berlin.de (K.F.H.); \\ maryke.fehlau@bionukleo.com (M.F.); sebastian.hans@tu-berlin.de (S.H.); peter.neubauer@tu-berlin.de (P.N.) \\ 2 BioNukleo GmbH, Ackerstraße 76, 13355 Berlin, Germany \\ * Correspondence: anke.wagner@tu-berlin.de \\ + K.F.H. and M.F. contributed equally to this work.
}

Citation: Hellendahl, K.F.; Fehlau, M.; Hans, S.; Neubauer, P.; Kurreck, A. Semi-Automated High-Throughput Substrate Screening Assay for Nucleoside Kinases. Int. J. Mol. Sci. 2021, 22, 11558. https://doi.org/ $10.3390 /$ ijms222111558

Academic Editor:

Roland Wohlgemuth

Received: 29 September 2021

Accepted: 20 October 2021

Published: 26 October 2021

Publisher's Note: MDPI stays neutral with regard to jurisdictional claims in published maps and institutional affiliations.

Copyright: (c) 2021 by the authors. Licensee MDPI, Basel, Switzerland. This article is an open access article distributed under the terms and conditions of the Creative Commons Attribution (CC BY) license (https:// creativecommons.org/licenses/by/ $4.0 /)$.
Abstract: Nucleoside kinases (NKs) are key enzymes involved in the in vivo phosphorylation of nucleoside analogues used as drugs to treat cancer or viral infections. Having different specificities, the characterization of NKs is essential for drug design and nucleotide analogue production in an in vitro enzymatic process. Therefore, a fast and reliable substrate screening method for NKs is of great importance. Here, we report on the validation of a well-known luciferase-based assay for the detection of NK activity in a 96-well plate format. The assay was semi-automated using a liquid handling robot. Good linearity was demonstrated $\left(\mathrm{r}^{2}>0.98\right)$ in the range of $0-500 \mu \mathrm{M}$ ATP, and it was shown that alternative phosphate donors like dATP or CTP were also accepted by the luciferase. The developed high-throughput assay revealed comparable results to HPLC analysis. The assay was exemplarily used for the comparison of the substrate spectra of four NKs using 20 (8 natural, 12 modified) substrates. The screening results correlated well with literature data, and additionally, previously unknown substrates were identified for three of the NKs studied. Our results demonstrate that the developed semi-automated high-throughput assay is suitable to identify best performing NKs for a wide range of substrates.

Keywords: nucleoside kinase; nucleoside analogues; nucleotide; screening; high throughput; luminescent assay; HPLC; robot; biocatalysis; luciferase assay

\section{Introduction}

Nucleotides are essential biomolecules involved in all cellular processes as building blocks of nucleic acids, energy storage, cellular communication mediators, and cofactors for many enzymatic reactions. Furthermore, nucleotide analogues have a broad range of applications in molecular biology and medicine. For example, modified nucleotides are used to label nucleic acids in PCR [1], for fluorescent in situ hybridization (FISH) [2], or microarray [3] applications. In addition, they improve nuclease stability of aptamers [4] and small interfering RNAs [5,6]. The relevance of nucleoside 5'-triphosphates (NTPs) is also evident in mRNA vaccines, which have become increasingly important in the current COVID-19 pandemic [7]. By incorporating modified NTPs such as N1-methylpseudouridine triphosphates or pseudouridine triphosphates into therapeutic mRNAs, significantly less activation of the innate immune response compared to uridine is induced [8]. In addition, there is evidence that modified mRNA is translated more efficiently than unmodified mRNA.

Nucleoside analogues are widely used to treat cancer [9], viral [9], and bacterial [10] infections. However, nucleoside analogue drugs are usually active only in the nucleotide form. Therefore, the therapeutic efficacy largely depends on the intracellular phosphorylation of the nucleoside analogue to a $5^{\prime}$-triphosphate. In three consecutive steps, nucleoside analogues are phosphorylated by (deoxy)nucleoside kinases ((d)NKs), (deoxy)nucleoside 
monophosphate kinases, and nucleoside diphosphate kinases [11]. Thus, to assess the therapeutic potential of new compounds in advance, a high-throughput assay for nucleoside and nucleotide kinases would be of great value.

Besides the importance in drug design, recombinant nucleoside and nucleotide kinases are valuable biocatalysts to produce modified nucleotides in enzymatic in vitro processes [12]. Enzymatic approaches offer an interesting alternative to the chemical synthesis by the use of mild reaction conditions, having a high regio- and stereoselectivity, and avoiding protection and deprotection steps [13,14].

Due to the importance of nucleoside and nucleotide kinases, the characterization of their substrate spectra and the identification of the most efficient phosphate donor is highly relevant. Therefore, different methods were developed to determine kinase activity [15]. Assays applicable towards a wide range of natural and modified substrates and phosphate donors include radiometric and chromatographic (TLC, HPLC) methods. However, these assays are time-consuming and hardly suitable for high-throughput applications. In addition, they would have to be optimized for some substrates. Other assays focus on the detection of adenosine $5^{\prime}$-triphosphate (ATP) consumption or production since ATP is the most commonly used phosphate donor for various kinases [16]. Assays based on lactate dehydrogenase, pyruvate oxidase, malachite green, pyruvate kinase, fluorescence polarization, FRET, or ELISA use the principle of measuring colorimetric or fluorometric changes $[17,18]$. Although these assays offer the opportunity to be used in high throughput, they are often expensive (especially if an antibody is involved) and require multi-step enzyme cascades. While the latter is not a concern when studying protein kinases, it is challenging for reactions catalyzed by nucleoside or nucleotide kinases because there is a higher risk that the non-natural nucleotides interfere with ATP detection $[17,18]$.

In contrast, bioluminescence-based assays using a luciferase to detect ATP are fast, high-throughput compatible, and based on only one enzyme [17,19]. In these assays, kinase activity is determined in two successive reactions. First, the kinase of interest catalyzes the transfer of a phosphate from a phosphate donor (mainly ATP) to the respective substrate. Afterwards, the residual phosphate donor is converted into a luminescence signal by a luciferase (Scheme 1). Thus, the kinase activity inversely corresponds to the residual phosphate donor concentration. Due to the advantages, bioluminescence-based assays have already been applied successfully for the activity detection as well as for inhibition and kinetic studies of many kinases $[16,20]$, including nucleoside/nucleotide kinases [19,21-24]. We recently demonstrated the use of a bioluminescence-based assay for the identification of substrates and kinetics for the human deoxycytidine kinase (HsdCK) [19]. However, so far, this was a manual assay.

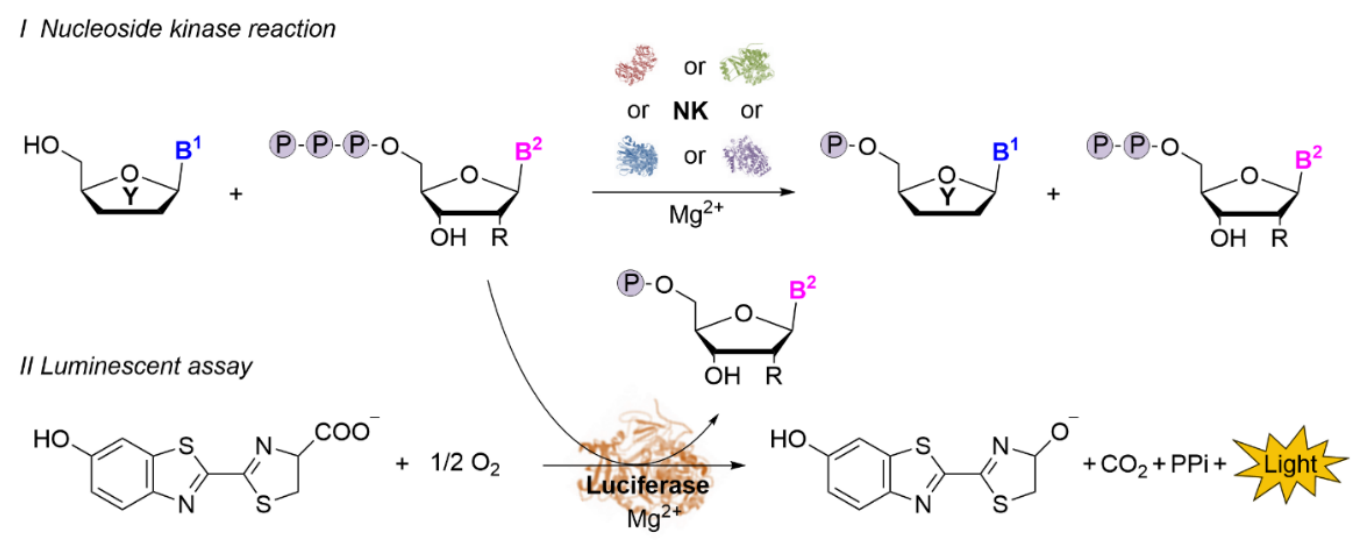

Scheme 1. Principle of the luminescent assay. The residual phosphate donor from the nucleoside kinase reaction (I) is detected by a luciferase through a luminescence signal (II). B ${ }^{1}$ and $\mathrm{B}^{2}$-nucleobase; $\mathrm{R}-\mathrm{H}$ or $\mathrm{OH}$; $\mathrm{Y}$-natural or modified sugar moiety. 
Here, we adapt this assay to be used on a robotic platform, so it can be applied as a fast, quantitative, and reliable semi-automated assay to analyze the phosphorylation of natural and modified nucleosides by nucleoside kinases using luciferase-based ATP detection. The accuracy of the assay was comparable to HPLC analytics. The advantage of this assay is demonstrated for the characterization of the substrate spectrum of four (d)NKs towards 20 nucleosides in a 96-well plate format. Autoluminescence or luciferase inhibition by the natural and modified nucleosides were not observed.

\section{Results and Discussion}

\subsection{Establishment of the Luminescent Assay with (Deoxy)NTP Standards}

To develop a high-throughput assay for the evaluation of the substrate spectrum of (d)NKs, the commercially available Kinase-Glo Max Luminescent Kinase Assay was chosen because it is described to exhibit high linearity up to an ATP concentration of $500 \mu \mathrm{M}$. The assay composition recommended by the manufacturer was slightly adjusted to reach volumes suitable for the 96-well plate format as previously described (for further information, see the materials and methods section) [19].

Initially, we were interested in whether the Ultra-Glo luciferase accepts other (deoxy)NTPs next to ATP since a wide range of phosphate donors are accepted by (d)NKs $[19,25,26]$. For that, standards of $500 \mu \mathrm{M}$ ATP, $2^{\prime}$-deoxyadenosine $5^{\prime}$-triphosphate (dATP), GTP, 2'-deoxyguanosine $5^{\prime}$-triphosphate (dGTP), cytidine $5^{\prime}$-triphosphate (CTP), $2^{\prime}$-deoxycytidine $5^{\prime}$-triphosphate (dCTP), uridine $5^{\prime}$-triphosphate (UTP), and thymidine $5^{\prime}$-triphosphate (TTP) were tested as substrates for the recombinant Ultra-Glo luciferase (Figure 1A). The highest luminescence signals were detected for ATP with values of up to 57.892 relative light units (RLU) (Figure 1B). This observation is in good accordance with literature data where a high specificity of luciferases towards ATP was described [27]. However, we also observed the acceptance of additional (deoxy)NTPs as substrates of the UltraGlo luciferase. The luminescence signal decreased in the order ATP $>>$ dATP (1128 RLU) > GTP (504 RLU) > UTP (220 RLU) > CTP (161 RLU) > dGTP, dCTP, TTP (<30 RLU). Except for dATP, the Ultra-Glo luciferase showed a lower preference for dNTPs and pyrimidine nucleotides compared to NTPs and purine nucleotides, respectively. To study the applicability of ATP, dATP, GTP, CTP, and UTP in the luminescent assay in more detail, standard curves in the range of 0-500 $\mu \mathrm{M}$ were prepared and revealed high accuracy with $\mathrm{r}^{2}$ values $>0.98$ (Figure 1C). Hence, except for dGTP, dCTP, and TTP, all natural (deoxy)NTPs are suitable substrates for the recombinant Ultra-Glo luciferase. This observation widens the applicability of the luminescence-based assay to study nucleoside and nucleotide kinases.

\subsection{Establishment of the Luciferase-Based (d)NK Activity Assay Using Natural (Deoxy)Nucleosides as Substrates}

In a first attempt to establish the NK activity assay, the influence of the nucleoside substrates on the luminescence signal was analyzed since it could not be excluded that certain nucleosides or nucleoside $5^{\prime}$-monophosphates (NMP) would affect the activity of the Ultra-Glo luciferase or show autoluminescence themselves. Therefore, we measured the luminescence of $400 \mu \mathrm{M}$ ATP alone or together with $400 \mu \mathrm{M}$ standards of eight natural (deoxy)nucleosides (adenosine (Ado), $2^{\prime}$-deoxyadenosine (dAdo), guanosine (Guo), 2'-deoxyguanosine (dGuo), cytidine (Cyd), 2'-deoxycytidine (dCyd), uridine (Urd), and thymidine (Thd)) and the respective NMPs ( $2^{\prime}$-deoxyadenosine $5^{\prime}$-monophosphate (dAMP), guanosine $5^{\prime}$-monophosphate (GMP), 2'-deoxyguanosine $5^{\prime}$-monophosphate (dGMP), cytidine $5^{\prime}$-monophosphate (CMP), 2'-deoxycytidine $5^{\prime}$-monophosphate (dCMP), uridine $5^{\prime}$-monophosphate (UMP), and thymidine $5^{\prime}$-monophosphate (TMP)) in NK reaction buffer (Table S1). The impact of adenosine $5^{\prime}$-monophosphate (AMP) was studied in more detail, as inhibitory effects on luciferases were described before [28]. To mimic the conditions of the activity assay, mixtures consisting of different AMP-ATP ratios were analyzed with a total concentration of $400 \mu \mathrm{M}$ (Figure S1). For all combinations, no significant influence on the luminescence signal was observed (Table S1, Figure S1). Hence, 
autoluminescence and luciferase inhibition were excluded for all tested (deoxy)nucleosides and (deoxy)NMPs.

A

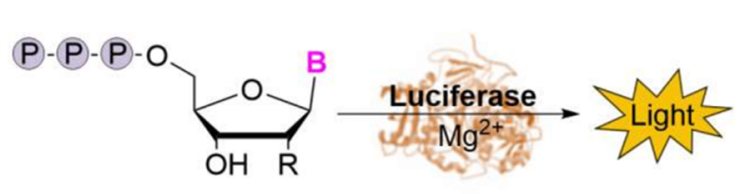

B

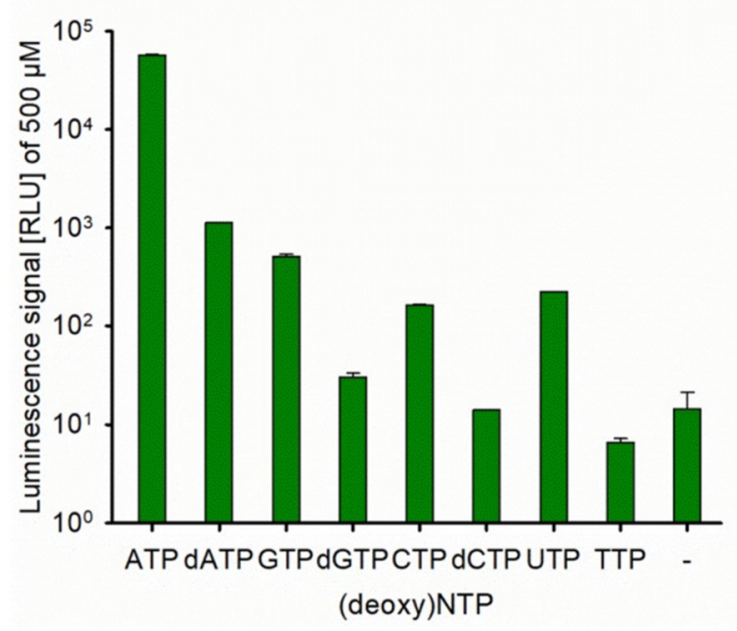

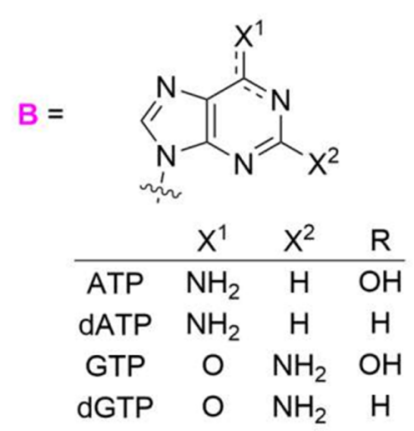

C

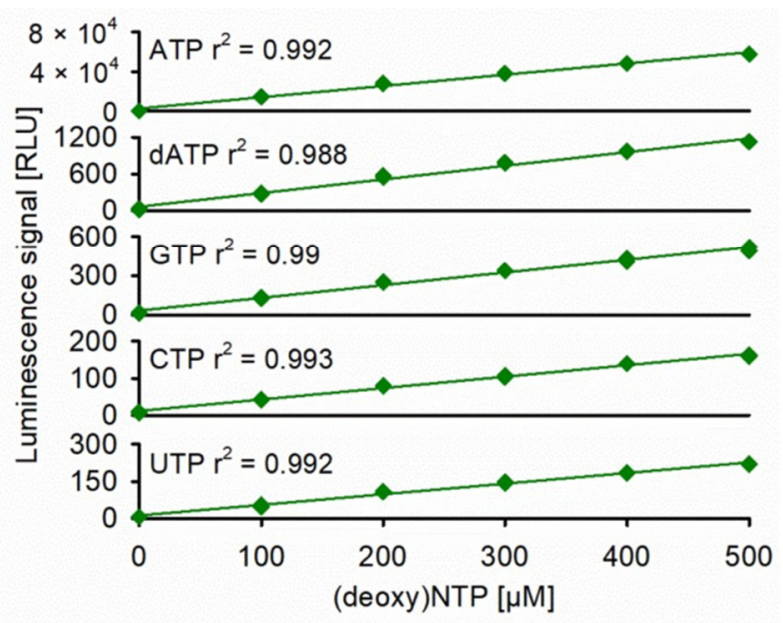

Figure 1. Validation of the acceptance of all natural (deoxy)NTPs as substrates for the Ultra-Glo luciferase using the luminescence-based assay. (A) Scheme of the luciferase reaction and structures of the applied nucleoside $5^{\prime}$-triphosphate substrates. (B) The acceptance of (deoxy)NTPs as substrates for the Ultra-Glo luciferase was analyzed with $500 \mu \mathrm{M}$ (deoxy)NTP in NK reaction buffer. Mean of the duplicates and standard deviation are indicated. (C) The linearity of the assay was evaluated with standards containing 0-500 $\mu \mathrm{M}$ ATP, dATP, GTP, CTP, or UTP. For the luminescent assay, $10 \mu \mathrm{L}$ phosphate donor standard was mixed with $80 \mu \mathrm{L}$ deionized water before adding $10 \mu \mathrm{L}$ of Kinase-Glo reagent. -: negative control (0 mM (deoxy)NTP).

To validate the accuracy of the assay, NK reactions were performed with human deoxycytidine kinase (HsdCK), ATP as phosphate donor and eight natural (deoxy)nucleosides (Ado, dAdo, Guo, dGuo, Cyd, dCyd, Urd, Thd, Figure 2A) at $37^{\circ} \mathrm{C}$ for $19 \mathrm{~h}$. Afterwards, ATP consumption and NMP formation were analyzed using the luminescence-based assay and data were compared to standard HPLC analytics. Using the luminescence-based assay, NMP formation was calculated based on ATP consumption. The determined NMP formation (Figures 2B and S2) and ATP consumption (Figures 2C and S2) were very comparable for both methods used. The deviations between both methods were between 0.49 and $7.53 \%$ (Table S2), with the difference being highest at low (deoxy)NMP formation. For Ado, Guo, and Urd, there was up to $9 \%$ ATP consumption detected by the luminescent assay, while no or only very little NMP formation was observed by HPLC. The susceptibility of the assay to error has been observed previously [16]. In order to avoid false-positive results at conversions $<10 \%$, it is therefore advisable to either (i) check product formation via HPLC or (ii) repeat the reaction with increased enzyme concentration to verify the substrate's acceptance. In general, the assay should be used preferentially to find the best performing NK showing significant substrate conversion $>50 \%$. 
In the next step, the substrate spectrum of $H s \mathrm{dCK}$ was evaluated and compared to literature data. HsdCK well accepted dAdo, dCyd, dGuo, and Cyd with (deoxy)NMP formation being above $80 \%$ (Figure 2B, Table S2). While the acceptance of dAdo, dCyd, and dGuo has already been described several times in the literature [19,29], reports on the acceptance of ribonucleotides are rare. Yet, the use of cytidine as substrates for human T-lymphoblast deoxycytidine kinase has been described before [30].

A
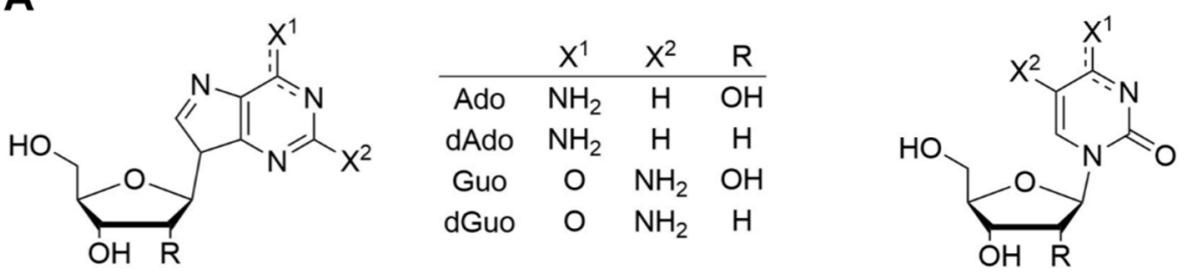

\begin{tabular}{cccc} 
& $\mathrm{X}^{1}$ & $\mathrm{X}^{2}$ & $\mathrm{R}$ \\
\hline Cyd & $\mathrm{NH}_{2}$ & $\mathrm{H}$ & $\mathrm{OH}$ \\
dCyd & $\mathrm{NH}_{2}$ & $\mathrm{H}$ & $\mathrm{H}$ \\
Urd & $\mathrm{O}$ & $\mathrm{H}$ & $\mathrm{OH}$ \\
Thd & $\mathrm{O}$ & $\mathrm{CH}_{3}$ & $\mathrm{H}$
\end{tabular}

B

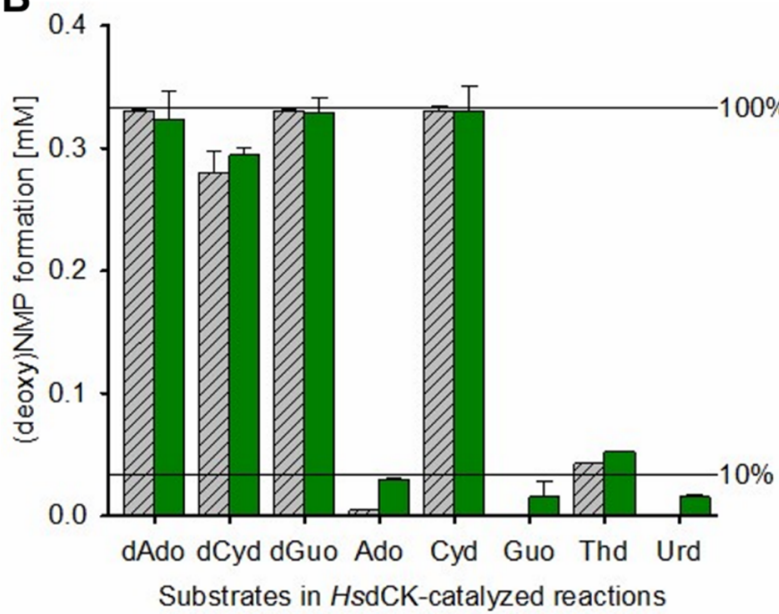

C

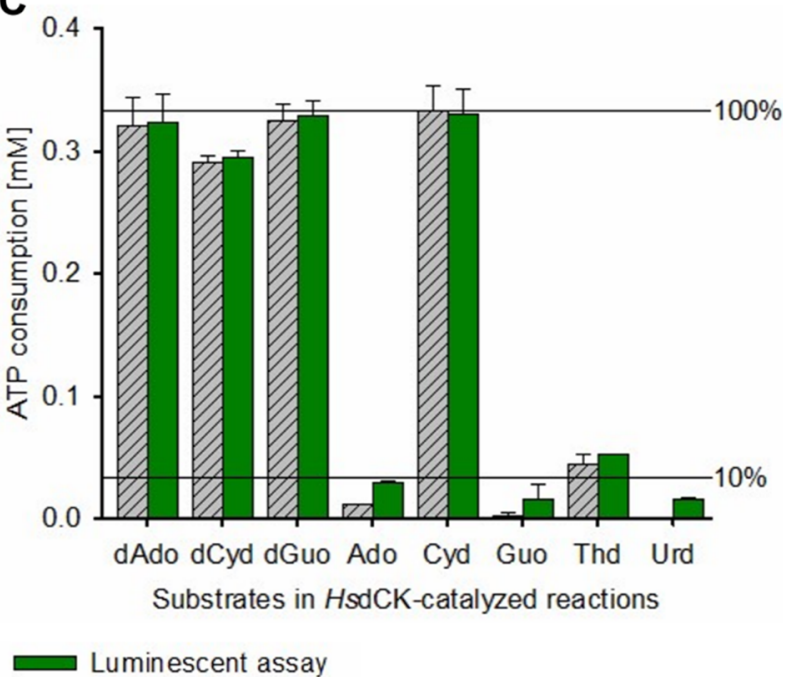

Figure 2. Validation of the luminescent assay for NK-catalyzed reactions. Natural nucleosides (A) were tested as substrates for HsdCK. HPLC analysis and the luminescent assay were used for the determination of (deoxy)NMP formation (B) and determination of ATP consumption (C). Reactions were performed with $0.33 \mathrm{mM}$ substrate and $0.4 \mathrm{mM}$ ATP in $70 \mathrm{mM}$ Tris- $\mathrm{HCl}$ [pH 7.6] supplemented with $5 \mathrm{mM} \mathrm{DTT}, 10 \mathrm{mM} \mathrm{MgCl}_{2}$, and $0.0002 \mathrm{U} \mathrm{HsdCK}$ at $37^{\circ} \mathrm{C}$ for $19 \mathrm{~h}$. Mean of the duplicates and standard deviation are indicated. For the luminescent assay, the (deoxy)NMP formation was calculated from the detected ATP consumption.

In this study, we also observed a minor accumulation of TMP ( 13\%, HPLC analysis) and AMP ( 1\%, HPLC analysis) with HsdCK as biocatalyst (Figure 2). There have been many reports that HsdCK does not accept thymidine, and it is believed that phosphorylation of this nucleoside in vivo is carried out by human thymidine kinase 1 (TK1) [31]. However, kinetic studies on wild-type and mutant HsdCK revealed that thymidine is accepted with low-rate constants [32], which correlates well with observations made in this study. The acceptance of adenosine as a substrate, however, has not been described so far. Although binding of adenosine to HsdCK was shown [33], AMP-forming activity has never been reported. Most probably, AMP appears due to minor degradation of ATP during the kinase reaction, as low AMP formation was also observed in controls lacking enzymes [34].

\subsection{Development of a High-Throughput Semi-Automated Activity Assay for Nucleoside Kinases}

A semi-automated approach using a liquid handling robot was developed to apply the luciferase-based assay in a highly accurate substrate screening for nucleoside kinases (Figure 3A, Scheme S1). Scripts were written to pipette the kinase reaction by the robot into 96-well PCR plates. Afterwards, the plates were manually transferred to an incubator. After 
completion of the reaction, plates were manually transferred back to the deck of the robot, and the luciferase reaction was pipetted. Subsequently, the assay plate was automatically transported to the plate reader to measure luminescence after $10 \mathrm{~min}$ incubation in the dark. Please see Table S3 and the externally hosted Supporting Information [34] for more detailed information on the approach.

To assess the accuracy of both the kinase reaction and the luminescent assay independently, the pipetting steps performed by the robot were evaluated by using dyes. The dyes were chosen to allow for a differentiation of the influence of the individual components on the overall reaction accuracy (for more information, see the Materials and Methods section). Robot pipetting accuracy was very high for both the kinase and the luciferase-catalyzed reaction (Figure S3).

A

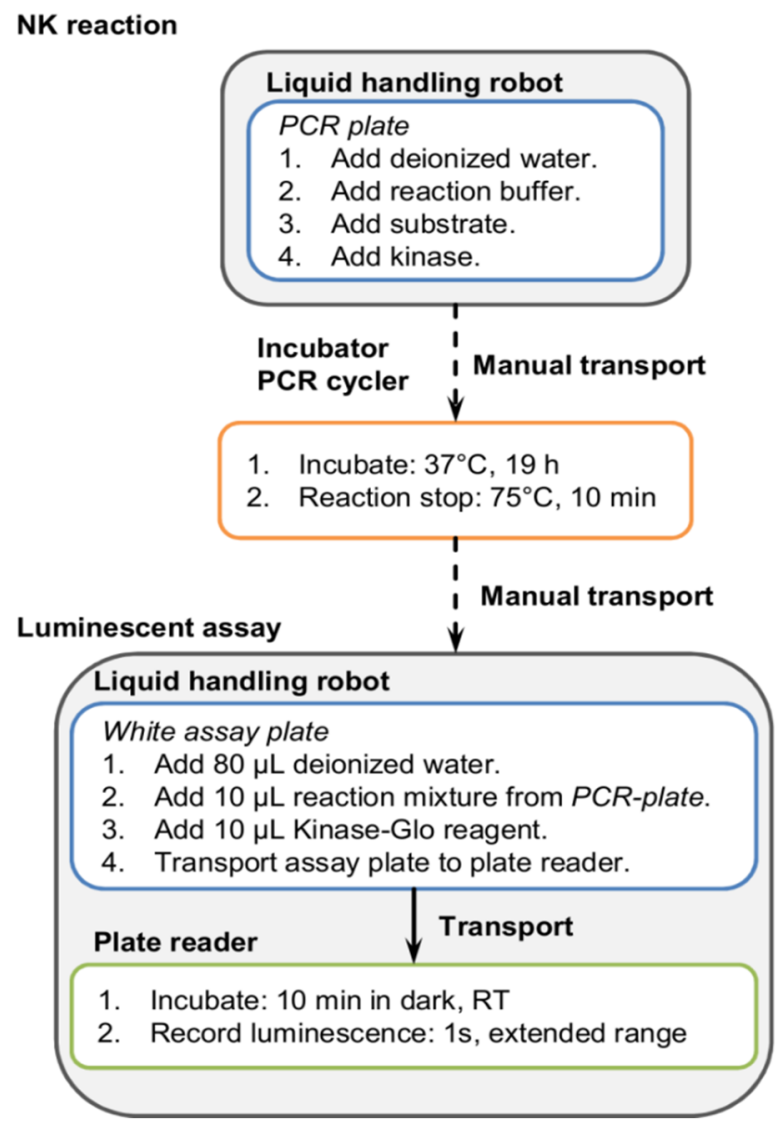

B

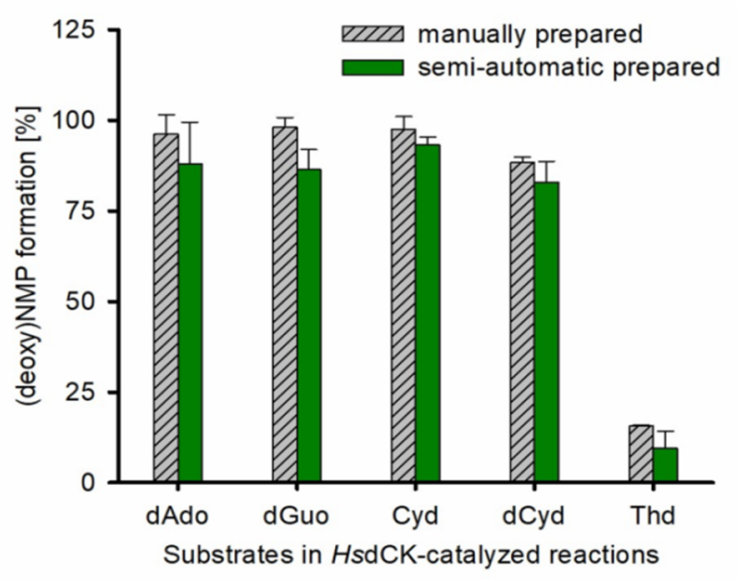

Figure 3. Semi-automated activity assay for nucleoside kinases. (A) The kinase reaction and the luminescent assay was automatically prepared by a liquid handling robot. A manual step was implemented to transport the reaction to an incubator and, after reaction complementation, back to the liquid handling robot. (B) HsdCK-catalyzed reactions with ATP as phosphate donor were manually and semi-automated prepared and analyzed using the luminescent assay. Mean of the duplicates and standard deviations are indicated.

HsdCK was chosen as a biocatalyst to validate the established approach in comparison to reactions that were pipetted manually. Kinase reactions were performed with natural (deoxy)nucleosides that were well accepted by HsdCK before (Figure 2B, dAdo, dGuo, Cyd, $\mathrm{dCyd}$, and Thd). The results of the semi-automated assay were in good accordance with manually pipetted reactions for all substrates tested (Figure 3B). 


\subsection{Application of the High-Throughput Semi-Automated Luminescent Assay to Compare the Substrate Spectra of Four NKs}

With the semi-automated high throughput screening assay in hand, the substrate spectra of three commercially available (d)NKs (HsdCK, human adenosine kinase (HsAK), and a viral thymidine kinase (TK)) were compared to the wide-spectrum Drosophila melanogaster dNK (DmdNK). In addition to eight natural nucleosides, 12 non-natural derivatives were included in the substrate screening assay. A set of sugar-modified (vidarabine (AraA), gemcitabine (dFdC), ganciclovir (GCV), acyclovir (ACV), 1-(2'-deoxy-2'-fluoro- $\beta$ D-arabinofuranosyl) uracil (FanaU)), base-modified (cladribine (CldA), 2-fluoroadenosine (FAdo), 5-fluorocytidine (FCyd), 5-ethynyl-2'-deoxyuridine (EdU)) as well as sugar- and base-modified nucleoside substrates (clofarabine (ClFanaA), fludarabine (FAraA), lamivudine (3TC)) were chosen (Figure 4A). To avoid false-positive results, activities $<10 \%$ were verified via HPLC, or the reactions were repeated with higher enzyme concentrations.

A

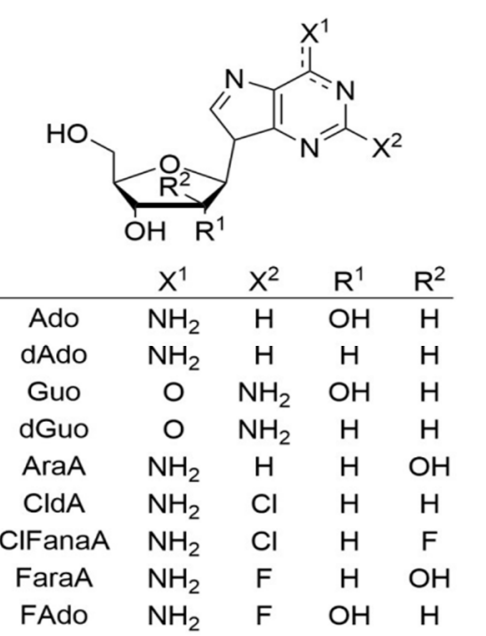

B

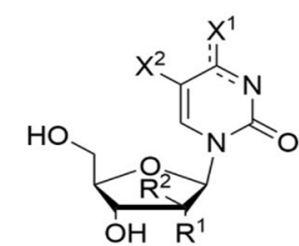

\begin{tabular}{ccccc} 
& $\mathrm{X}^{1}$ & $\mathrm{X}^{2}$ & $\mathrm{R}^{1}$ & $\mathrm{R}^{2}$ \\
\hline Cyd & $\mathrm{NH}_{2}$ & $\mathrm{H}$ & $\mathrm{OH}$ & $\mathrm{H}$
\end{tabular}

dCyd $\mathrm{NH}_{2} \quad \mathrm{H} \quad \mathrm{H} \quad \mathrm{H}$

Urd $\mathrm{O} \quad \mathrm{H} \quad \mathrm{OH} \quad \mathrm{H}$

Thd $\mathrm{O} \quad \mathrm{CH}_{3} \mathrm{H} \quad \mathrm{H}$

$\begin{array}{lllll}\text { dFdC } & \mathrm{NH}_{2} & \mathrm{H} & \mathrm{F} & \mathrm{F}\end{array}$

FanaU $\mathrm{O} \quad \mathrm{H} \quad \mathrm{H} \quad \mathrm{F}$

FCyd $\mathrm{NH}_{2} \quad \mathrm{~F} \quad \mathrm{OH} \quad \mathrm{H}$

EdU $\stackrel{\mathrm{CCH}}{\mathrm{O}} \mathrm{H}$<smiles>Nc1ccn(C2COCC2CO)c(=O)n1</smiles><smiles>Nc1nc2c(ncn2COC(CO)CO)c(=O)[nH]1</smiles>

GCV<smiles>Nc1nc(=O)c2ncn(COCCO)c2[nH]1</smiles>

3TC

(1)

ACV

\section{C}

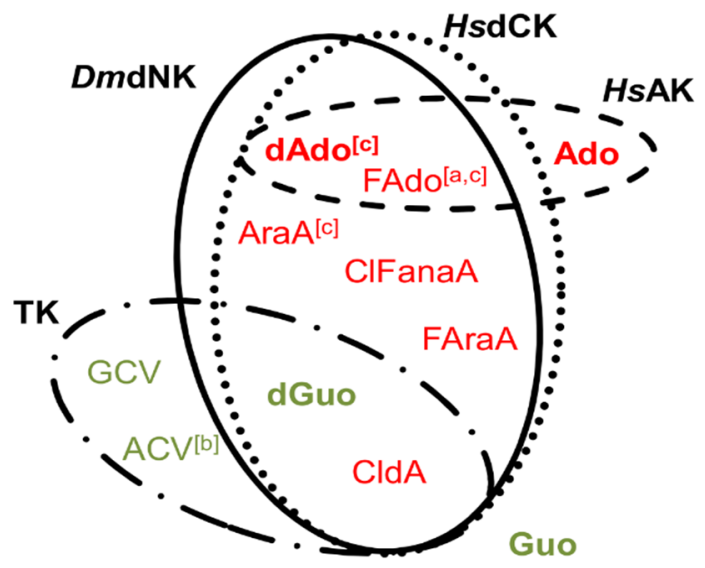

Ado and derivatives

Guo and derivatives

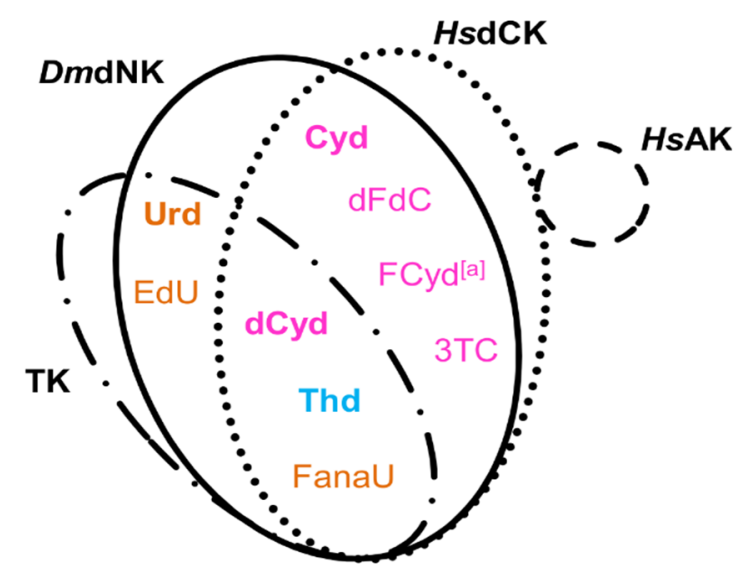

Cyd and derivatives

Urd and derivatives

Thd and derivatives

Figure 4. Semi-automated substrate screening for DmdNK, HsdCK, HsAK, and TK. The substrate spectra of the four (deoxy)nucleoside kinases were tested with 20 nucleosides (A). Activities with purine (B) and pyrimidine (C) nucleoside substrates were determined for the chosen NKs using standard screening conditions. If a discrepancy to literature data was observed, assay conditions were optimized: [a] $0.004 \mathrm{U}$ DmdNK were applied, [b] $0.0012 \mathrm{U}$ TK were used, [c] HsAK reactions including $50 \mathrm{mM} \mathrm{KCl}$ and $0.004 \mathrm{U}$ enzyme (see also Figure 5). Substrates were only presented if conversions $>10 \%$ were observed. Please see Table S4 for the conversions. 
In literature, DmdNK and HsdCK were described as wide-spectrum enzymes [19,35-39]. Indeed, both enzymes showed a very comparable broad substrate spectrum with the compounds tested. Next to dAdo, dGuo, and Thd, both enzymes accepted diverse baseand sugar-modified nucleosides such as AraA, CldA, ClFanaA, FAraA, FAdo, dFdC, FanaU, FCyd, and 3TC (Figure 4B,C). Both DmdNK and HsdCK preferred deoxy- and arabinonucleosides over ribonucleosides. Open-ring derivatives GCV and ACV were accepted by neither DmdNK nor HsdCK. One difference between the two enzymes is the acceptance of uridine and its derivatives. While DmdNK accepted uridine and EdU, this was not the case for HsdCK. Whereas most observations correspond well with literature data available for HsdCK $[11,37,40,41]$ or DmdNK [12,35-37,42], an activity towards a few substrates was not known before. This concerns the acceptance of FAdo, FanaU, and FCyd by HsdCK and activities towards ClFanaA, FanaU, EdU, and 3TC by DmdNK.

Although viral thymidine kinases are known to accept a broad spectrum of nucleoside analogues [11], the tested TK displayed a narrower substrate spectrum compared to $D m \mathrm{dNK}$ or HsdCK (Figure 4B,C). The screening mainly revealed activities towards deoxyor arabino-nucleoside substrates. Not only pyrimidines but also some purine nucleoside analogues were converted by TK, although to a much lower extent than by $D m \mathrm{dNK}$ and HsdCK. Interestingly, unlike DmdNK and HsdCK, TK phosphorylates the open-ring guanosine analogues GCV and ACV, which is in good agreement with literature data on viral TKs [11]. The ability of a viral TK to phosphorylate CldA and FanaU was shown for the first time in this study.

Compared to the other three analyzed enzymes, HsAK appears to be the most specific kinase. Here, this enzyme only showed activity for the natural purine nucleoside Ado. However, HsAK seems to be more promiscuous. Previous studies by Yamada et al. showed phosphorylation of dAdo and AraA with AK from the human liver [25], and we showed the conversion of FAdo before [12]. Both studies relied on optimized conditions to reach significant conversions, e.g., the addition of potassium chloride $(\mathrm{KCl})$ was described to be beneficial for HsAK activity [25], something which could be analyzed with the here described assay.

\subsection{Application of the High-Throughput Semi-Automated Assay to Optimize Reaction Conditions for HsAK}

To validate the impact of optimized reaction conditions on the activity of HsAK, the semi-automated assay was applied. Reactions with potassium addition and/or an increased enzyme concentration were analyzed and compared to the standard conditions.

While the addition of $50 \mathrm{mM} \mathrm{KCl}$, did not improve NMP formation, a 20-fold increase in HsAK led to an increased substrate conversion of dAdo, AraA, and FAdo (Figure 5). A combination of $\mathrm{KCl}$ addition and increased enzyme concentration further improved NMP formation for dAdo and FAdo. In accordance with literature data, no significant conversion of AraA was observed, which confirms the results by Yamada et al., who showed HsAK activity towards AraA to be max. 7\% compared to Ado under optimized conditions [25].

Higher enzyme concentrations might be applied in future screenings to avoid overlooking small activities towards single nucleoside analogues. In this context, the example of HsAK revealed that variations in reaction conditions might be necessary to ensure optimal conditions for each enzyme. 


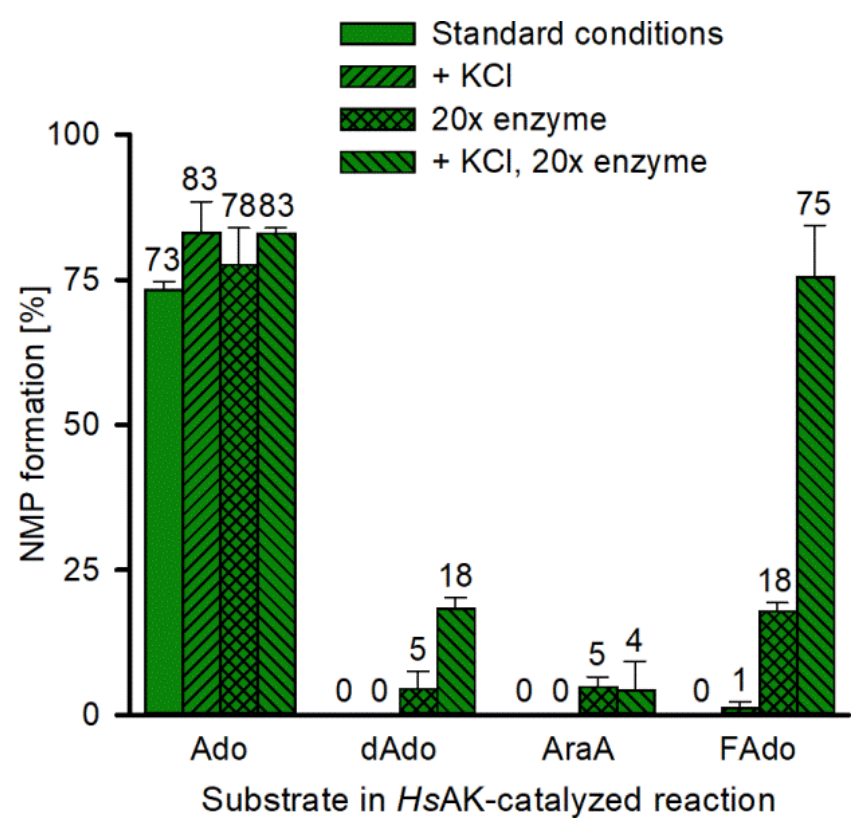

Figure 5. HsAK activity for Ado, dAdo, AraA, and FAdo. Standard screening conditions are compared to optimized conditions ( $\mathrm{KCl}$ addition and/or 20-fold increase of enzyme concentration). Reactions were performed with $0.33 \mathrm{mM}$ substrate and $0.4 \mathrm{mM}$ ATP in $70 \mathrm{mM}$ Tris- $\mathrm{HCl}$ [pH 7.6] supplemented with $5 \mathrm{mM} \mathrm{DTT}, 10 \mathrm{mM} \mathrm{MgCl}, 0 / 50 \mathrm{mM} \mathrm{KCl}$, and $0.0002 / 0.004 \mathrm{U} \mathrm{HsdCK}$ at $37^{\circ} \mathrm{C}$ for $19 \mathrm{~h}$. Mean of the duplicates and standard deviations are indicated. The NMP formation was calculated from the detected ATP consumption.

\section{Materials and Methods}

\subsection{General Remarks}

All chemicals and solvents were of analytical grade or higher and purchased, if not stated otherwise, from Sigma-Aldrich (Steinheim, Germany), Carl Roth (Karlsruhe, Germany), Fluka Chemie GmbH (Buchs, Switzerland), PanReac AppliChem (Darmstadt, Germany), or VWR (Darmstadt, Germany). Nucleosides and nucleotides were acquired from Alfa Aesar (Kandel, Germany), Carl Roth (Karlsruhe, Germany), Sigma-Aldrich (Steinheim, Germany), Carbosynth Limited (Berkshire, United Kingdom), and TCI (Eschborn, Germany). Stock solutions with concentrations of 100,10, or $1 \mathrm{mM}$ were prepared in deionized water, and aliquots were stored at $-20^{\circ} \mathrm{C}$.

The human adenosine kinase (HsAK, NK14), human deoxycytidine kinase (HsdCK, NK13), and viral thymidine kinase (TK, NK16) were obtained from BioNukleo GmbH (Berlin, Germany) and stored at $-20{ }^{\circ} \mathrm{C}$.

The Drosophila melanogaster deoxynucleoside kinase (DmdNK) plasmid was kindly provided by Prof. Birgitte Munch-Petersen (Roskilde University).

\subsection{Expression of DmdNK}

DmdNK was produced as described before [36]. Briefly, it was expressed as a GSTfusion protein in Escherichia coli BL21 and purified using glutathione sepharose. The GST tag was cleaved off using thrombin, and the enzyme was stored in aliquots at $-20{ }^{\circ} \mathrm{C}$, including 50\% glycerol, $1 \%$ Triton X-100, and $1 \mathrm{mM}$ dithiothreitol (DTT).

\subsection{The Luminescent Assay}

The Kinase-Glo Max assay (Promega, Madison/Wisconsin, USA) was used to detect (deoxy)NTPs in 96-well white assay plates (Costar 655094, Greiner Bio-One, Kremsmünster, Austria) as previously described [19]. Briefly, the assay was performed with $10 \mu \mathrm{L}$ sample/standard and $10 \mu \mathrm{L}$ Kinase-Glo reagent following the 1:1 ratio suggested by the manufacturer. The total assay volume was adapted for the use in 96-well plate format to $100 \mu \mathrm{L}$ by adding $80 \mu \mathrm{L}$ deionized water. Luminescence was measured in a Synergy ${ }^{\circledR}$ 
Mx Multi-Mode Microplate Reader (Bio Tek Instruments, Winooski, VT, USA) for $1 \mathrm{~s}$ and extended dynamic range after $10 \mathrm{~min}$ incubation in the dark.

Unless otherwise stated, each standard was tested twice and each sample three times.

\subsection{Detection of (Deoxy)NTP and Mixed ATP Standards}

The acceptance of (deoxy)NTPs as substrates for the Ultra-Glo luciferase was analyzed by the preparation of standards ranging from 0 to $500 \mu \mathrm{M}$ of ATP, UTP, GTP, CTP, dATP, TTP, dGTP, and dCTP in NK reaction buffer (70 mM Tris [pH 7.6], $10 \mathrm{mM} \mathrm{MgCl}_{2}, 5 \mathrm{mM}$ DTT).

Autoluminescence and possible luciferase inhibition were tested with $400 \mu \mathrm{M}$ ATP alone or together with $400 \mu \mathrm{M}$ of Ado, dAdo, Guo, dGuo, Cyd, dCyd, Urd, or Thd in NK reaction buffer, respectively.

To analyze the impact of AMP, standards in NK buffer with $0.4 \mathrm{mM}$ ATP, $0.3 \mathrm{mM}$ ATP/0.1 mM AMP, $0.2 \mathrm{mM}$ ATP/0.2mM AMP, $0.1 \mathrm{mM}$ ATP/0.3 mM AMP, and $0.4 \mathrm{mM}$ AMP were used.

\subsection{Nucleoside Kinase Reactions}

The NK reactions were performed in a total volume of $150 \mu \mathrm{L}$ consisting of $0.4 \mathrm{mM}$ ATP, $0.33 \mathrm{mM}$ nucleoside and $0.0002 \mathrm{U}$ enzyme in NK reaction buffer $(70 \mathrm{mM}$ Tris [pH 7.6], $10 \mathrm{mM} \mathrm{MgCl} 2,5 \mathrm{mM}$ DTT). Reaction mixtures were incubated at $37^{\circ} \mathrm{C}$ for $19 \mathrm{~h}$ and stopped by a heating step at $75^{\circ} \mathrm{C}$ for $10 \mathrm{~min}$.

Optimized reaction conditions included $6 \times(0.0012 \mathrm{U}$ for TK $)$ or $20 \times(0.004 \mathrm{U}$ for $D m \mathrm{dNK}$ and HsAK) increased enzyme concentration and/or the addition of $50 \mathrm{mM}$ $\mathrm{KCl}$ (HsAK).

The following controls were prepared on the same assay plate as the reactions to ensure the same incubation conditions: (a) negative control without enzyme, (b) substrate control without ATP, and (c) basal activity control without substrate.

To calculate the consumed ATP in the kinase reaction, the following Equation (1) was used, whereby $R$ is the average luminescence signal of the kinase reaction, $B$ is the difference of the negative control and the basal activity, $S$ is the substrate control, and $N$ is the average luminescence signal of the negative control:

$$
\text { Consumed } A T P_{\text {Lum }}[\%]=100-\left(100 \times \frac{R+B+S}{N+S}\right)
$$

Due to the molar ratio of ATP and substrate of 1.2:1, the (deoxy)NMP product formation was calculated by multiplying the consumed ATP with the factor 1.2 (Equation (2)).

$$
\text { Product }_{\text {Lum }}[\%]=\text { Consumed } A T P_{\text {Lum }} \times 1.2
$$

Values were restricted to $0-100 \%$.

\subsection{Semi-Automated Assay to Determine the Activity of Nucleoside Kinases}

Pipetting steps were performed by the liquid handling robot Microlab Star (Hamilton Bonaduz AG, Bonaduz, Switzerland). The reaction mixture was incubated for $19 \mathrm{~h}$ at $37^{\circ} \mathrm{C}$ in 96-well PCR plates (Greiner Bio-One 652290, Kremsmünster, Austria). The PCR plates were closed with EASYseal ${ }^{\mathrm{TM}}$ sealing film (Greiner Bio-One A5596) and arched auto-sealing lids (Bio-Rad, Hercules, CA, USA) preheated to $50^{\circ} \mathrm{C}$. The reaction was stopped by heating to $75{ }^{\circ} \mathrm{C}$ for $10 \mathrm{~min}$ with lid heat set to $85^{\circ} \mathrm{C}$ in a Primus 96 plus (MWG Biotech Inc, Ebersberg, Germany) PCR cycler. All kinases' reactions were performed in duplicates, and the luciferase assay was performed in triplicates.

\subsection{Accuracy of the Assay}

The accuracy of the assay was analyzed with colored solutions. For that, the kinase reaction PCR plate and the assay plate were prepared independently by the liquid handling robot. Then, the absorbance between 300 and $700 \mathrm{~nm}$ was recorded, and the differences 
in the spectra were evaluated. For the kinase reaction plate, the dyes blue (water), pink (buffer), green (substrate), and black (enzyme) were used. From the PCR plate, $100 \mu \mathrm{L}$ were manually transferred to an assay plate for analysis. For the assay plate, blue (water), pink (kinase reaction), and black (Kinase-Glo reagent) were applied.

The $Z^{\prime}$-value for each plate was calculated according to Equation (3) [16], whereby $S D_{\text {negative }}$ and $S D_{\text {substrate }}$ are the standard deviations, and Average negative $_{\text {and }}$ Average substrate are the means for the negative and the substrate controls, respectively.

$$
Z^{\prime} \text { value }=1-\frac{3 \times S D_{\text {negative }}+3 \times S D_{\text {substrate }}}{\text { Average }_{\text {negative }}-\text { Average }_{\text {substrate }}}
$$

\subsection{High-Performance Liquid Chromatography (HPLC)}

For HPLC analysis, $100 \mu \mathrm{L}$ of the kinase reaction was mixed with $100 \mu \mathrm{L} \mathrm{MeOH}$ and centrifuged at $4{ }^{\circ} \mathrm{C}$ and $21.500 \times g$ for $20 \mathrm{~min}$. Then, $75 \mu \mathrm{L}$ of the supernatant were added to $25 \mu \mathrm{L}$ deionized water, and the mixture was transferred to an HPLC vial with inlet. Samples were analyzed by HPLC-DAD (Agilent 1200 series, Santa Clara/California, USA) at $260 \mathrm{~nm}$ using a Kinetex Evo column (C18, 100 A, $250 \times 4.6$ mm, Phenomenex, Aschaffenburg, Germany) as described previously [12]. Briefly, a flow rate of $1 \mathrm{~mL} \mathrm{~min}^{-1}$, a separation temperature of $34{ }^{\circ} \mathrm{C}$, and a gradient of $\mathrm{A}\left(\mathrm{KH}_{2} \mathrm{PO}_{4} / \mathrm{K}_{2} \mathrm{HPO}_{4}: 0.1 \mathrm{M}\right.$, tetrabutylammonium bisulfate: $8 \mathrm{mM}, \mathrm{pH} 5.4)$ and $\mathrm{B}(70 \% \mathrm{~A}, 30 \%$ methanol) was applied: $0 \mathrm{~min}-80 \% \mathrm{~A}$, $4 \mathrm{~min}-80 \% \mathrm{~A}, 14 \mathrm{~min}-40 \% \mathrm{~A}, 35 \mathrm{~min}-36.5 \% \mathrm{~A}, 35.5 \mathrm{~min}-80 \% \mathrm{~A}$, and $38 \mathrm{~min}-80 \% \mathrm{~A}$.

Typical retention times [min] were for AMP - 8.2, ADP-15.4, and ATP-22.9. The retention times of the (deoxy)nucleosides and (deoxy)nucleoside monophosphates are given in Table S5.

The consumed ATP and formed product in the kinase reaction mix were calculated using the following Equations (4) and (5), whereby $A_{X}$ is the peak area of ATP, $A_{\text {total }}$ is the sum of all adenosine nucleotides (ATP, ADP, AMP), $P_{X}$ is the peak area of the produced $\mathrm{NMP}$, and $P_{\text {total }}$ is the sum of the substrate and product(s):

$$
\begin{gathered}
\text { Consumed ATP } P_{\text {HPLC }}[\%]=\left(1-\frac{A_{X}}{A_{\text {Total }}}\right) \times 100 \\
\text { Product }_{\text {HPLC }}[\%]=\frac{P_{X}}{P_{\text {total }}} \times 100
\end{gathered}
$$

\section{Conclusions}

The luciferase-based assay is very suitable to screen the activity of (d)NKs towards a wide range of nucleoside substrates. Interference of the nucleoside substrates or the NMP products with the luminescent assay was not observed. The results obtained in this study correlated very well with literature data. In addition, further previously unknown substrates were identified for HsdCK, DmdNK, and the viral TK. By automation, a nonradiometric, fast, inexpensive, reliable, and simple method was established, which shows comparable accuracy as standard HPLC analytics. In contrast to HPLC analytics, this assay allows the rapid and parallel analysis of large sample numbers. Additionally, the lower substrate and enzyme volumes required and the avoidance of solvents lead to reduced costs and improved sustainability.

The automated luciferase-based assay has great potential to be used to screen the substrate spectrum of other kinases involved in nucleotide metabolism such as NMP and NDP kinases or in inhibition/kinetic studies of nucleoside kinase-catalyzed reactions. Furthermore, the impact of varying phosphate donors on the activity of nucleoside or nucleotide kinases can be evaluated as the Ultra-Glo luciferase also accepts dATP, GTP, CTP, and UTP next to ATP. Future research will include the full automatization of the assay. Furthermore, it will be evaluated if the assay can be applied for enzyme cascade reactions to form NTPs from nucleosides. 
Supplementary Materials: Available online at https://www.mdpi.com/article/10.3390/ijms222 111558/s1 and https:/ / doi.org/10.5281/zenodo.5363310. Figure S1: Mixed ATP/AMP standards, Figure S2: Exemplary HPLC chromatograms for HsdCK-catalyzed reactions with natural substrates, Figure S3: Accuracy of the NK activity assay, Scheme S1: Semi-automated substrate screening assay for nucleoside kinases, Table S1: Autoluminescence and luciferase inhibition test with mixed ATP and nucleoside/NMP standards, Table S2: Comparison of HPLC and the luminescent assay for the determination of ATP consumption and (deoxy)NMP formation in HsdCK-catalyzed reactions, Table S3: Assay protocol, Table S4: Activities of four (deoxy)nucleoside kinases with 20 natural (upper part) and modified nucleoside substrates (lower part) were determined using the semi-automated high-throughput NK assay, Table S5: Typical retention times for the HPLC analysis.

Author Contributions: Conceptualization, K.F.H., M.F., P.N., and A.K.; data curation: K.F.H. and M.F.; formal analysis, K.F.H. and M.F.; funding acquisition, P.N. and A.K.; investigation, K.F.H. and M.F.; methodology, K.F.H. and M.F.; project administration, K.F.H., M.F., P.N., and A.K.; resources, P.N. and A.K.; software, K.F.H. and S.H.; supervision, P.N. and A.K.; validation, K.F.H. and M.F.; visualization, K.F.H., M.F., and A.K.; writing-original draft, K.F.H. and M.F.; writing-review and editing, K.F.H., M.F., S.H., P.N., and A.K. All authors have read and agreed to the published version of the manuscript.

Funding: K.F.H. was funded by the Deutsche Forschungsgemeinschaft (DFG, German Research Foundation)—grant number 392246628.

Institutional Review Board Statement: Not applicable.

Informed Consent Statement: Not applicable.

Data Availability Statement: All data depicted visually in the items and in the main text as well as in the Supplementary Material are available from an externally hosted Supporting Information [34].

Acknowledgments: We thank Florian Glauche for his assistance in robot programing and Lena Panse for her support in the laboratory. We are grateful to Birgitte Munch-Petersen for supplying Drosophila melanogaster deoxynucleoside kinase plasmid. We thank the Open Access Publishing funds of TU Berlin for the support of this publication.

Conflicts of Interest: A.K. is CEO, M.F., and P.N. are board members of the biotech company BioNukleo $\mathrm{GmbH}$. The authors declare no conflict of interest.

\begin{abstract}
Abbreviations
ACV: acyclovir, Ado: adenosine, AraA: vidarabine, ATP: adenosine 5'-triphosphate, CldA: cladribine, ClFanaA: clofarabine, CTP: cytidine $5^{\prime}$-triphosphate, Cyd: cytidine, dAdo: $2^{\prime}$-deoxyadenosine, dATP: $2^{\prime}$-deoxyadenosine 5-triphosphate, dCTP: $2^{\prime}$-deoxycytidine $5^{\prime}$-triphosphate, dCyd: $2^{\prime}$-deoxycytidine, dFdC: gemcitabine, dGTP: $2^{\prime}$-deoxyguanosine $5^{\prime}$-triphosphate, dGuo: $2^{\prime}$-deoxyguanosine, DmdNK: Drosophila melanogaster deoxynucleoside kinase, dNK: deoxynucleoside kinase, EdU: 5-ethynyl- $2^{\prime}$ deoxyuridine, FAdo: 2-fluoroadenosine, FanaU: 1-(2'-Deoxy-2'-fluoro- $\beta$-D-arabino- furanosyl) uracil, FAraA: fludarabine, FCyd:5-fluorocytidine, GCV: ganciclovir, GTP: guanosine 5 'triphosphate, Guo: guanosine, HsAK: Homo sapiens adenosine kinase, HsdCK: Homo sapiens deoxycytidine kinase, NK: nucleoside kinase, NMP: nucleoside- $5^{\prime}$-monophosphate, NTP: nucleoside-5'-triphosphate, RLU: relative light units, 3TC: lamivudine, Thd: thymidine, TK: thymidine kinase, TTP: thymidine $5^{\prime}$-triphosphate, Urd: uridine, UTP: uridine $5^{\prime}$-triphosphate
\end{abstract}

\title{
References
}

1. Zaccolo, M.; Williams, D.M.; Brown, D.M.; Gherardi, E. An approach to random mutagenesis of DNA using mixtures of triphosphate derivatives of nucleoside analogues. J. Mol. Biol. 1996, 255, 589-603. [CrossRef]

2. Silvia, F.; Joana, B.; Pedro, M.; Céu, F.; Jesper, W.; Filipe, A.N. Mismatch discrimination in fluorescent in situ hybridization using different types of nucleic acids. Appl. Microbiol. Biotechnol. 2015, 99, 3961-3969. [CrossRef]

3. Bumgarner, R. Overview of DNA Microarrays: Types, Applications, and Their Future. Curr. Protoc. Mol. Biol. 2013, 101, 22.1.1-22.1.11.

4. Gragoudas, E.S.; Adamis, A.P.; Cunningham, E.T.; Feinsod, M.; Guyer, D.R. Pegaptanib for neovascular age-related macular degeneration. N. Engl. J. Med. 2004, 351, 2805-2816. [CrossRef] 
5. Kalota, A.; Karabon, L.; Swider, C.R.; Viazovkina, E.; Elzagheid, M.; Damha, M.J.; Gewirtz, A.M. 2'-deoxy-2'-fluoro-beta-Darabinonucleic acid (2'F-ANA) modified oligonucleotides (ON) effect highly efficient, and persistent, gene silencing. Nucleic Acids Res. 2006, 34, 451-461. [CrossRef]

6. Watts, J.K.; Corey, D.R. Silencing disease genes in the laboratory and the clinic. J. Pathol. 2012, 226, 365-379. [CrossRef]

7. Granados-Riveron, J.T.; Aquino-Jarquin, G. Engineering of the current nucleoside-modified mRNA-LNP vaccines against SARS-CoV-2. Biomed. Pharm. 2021, 142, 111953. [CrossRef] [PubMed]

8. Pardi, N.; Hogan, M.J.; Porter, F.W.; Weissman, D. mRNA vaccines-A new era in vaccinology. Nat. Rev. Drug Discov. 2018, 17, 261-279. [CrossRef] [PubMed]

9. Jordheim, L.P.; Durantel, D.; Zoulim, F.; Dumontet, C. Advances in the development of nucleoside and nucleotide analogues for cancer and viral diseases. Nat. Rev. Drug Discov. 2013, 12, 447-464. [CrossRef]

10. Thomson, J.M.; Lamont, I.L. Nucleoside Analogues as Antibacterial Agents. Front. Microbiol. 2019, 10, 952. [CrossRef] [PubMed]

11. Deville-Bonne, D.; El Amri, C.; Meyer, P.; Chen, Y.; Agrofoglio, L.A.; Janin, J. Human and viral nucleoside/nucleotide kinases involved in antiviral drug activation: Structural and catalytic properties. Antiviral Res. 2010, 86, 101-120. [CrossRef]

12. Fehlau, M.; Kaspar, F.; Hellendahl, K.F.; Schollmeyer, J.; Neubauer, P.; Wagner, A. Modular Enzymatic Cascade Synthesis of Nucleotides Using a (d)ATP Regeneration System. Front. Bioeng. Biotechnol. 2020, 8, 854. [CrossRef]

13. Kore, A.; Srinivasan, B. Recent Advances in the Syntheses of Nucleoside Triphosphates. Curr. Org. Synth. 2014, 10, 903-934. [CrossRef]

14. Winkler, C.K.; Schrittwieser, J.H.; Kroutil, W. Power of Biocatalysis for Organic Synthesis. ACS Cent. Sci. 2021, 7, 55-71. [CrossRef] [PubMed]

15. Acker, M.G.; Auld, D.S. Considerations for the design and reporting of enzyme assays in high-throughput screening applications. Perspect. Sci. 2014, 1, 56-73. [CrossRef]

16. Baki, A.; Bielik, A.; Molnár, L.; Szendrei, G.; Keserü, G.M. A high throughput luminescent assay for glycogen synthase kinase-3 $\beta$ inhibitors. Assay Drug Dev. Technol. 2007, 5, 75-83. [CrossRef]

17. Ma, H.; Deacon, S.; Horiuchi, K. The challenge of selecting protein kinase assays for lead discovery optimization. Expert Opin. Drug Discov. 2008, 3, 607-621. [CrossRef]

18. Feng, J.; Chen, Y.; Pu, J.; Yang, X.; Zhang, C.; Zhu, S.; Zhao, Y.; Yuan, Y.; Yuan, H.; Liao, F. An improved malachite green assay of phosphate: Mechanism and application. Anal. Biochem. 2011, 409, 144-149. [CrossRef]

19. Hellendahl, K.F.; Kamel, S.; Wetterwald, A.; Neubauer, P.; Wagner, A. Human Deoxycytidine Kinase Is a Valuable Biocatalyst for the Synthesis of Nucleotide Analogues. Catalysts 2019, 9, 997. [CrossRef]

20. Singh, M.; Tiwari, P.; Arora, G.; Agarwal, S.; Kidwai, S.; Singh, R. Establishing Virulence Associated Polyphosphate Kinase 2 as a drug target for Mycobacterium tuberculosis. Sci. Rep. 2016, 6, 26900. [CrossRef]

21. Helenius, M.; Jalkanen, S.; Yegutkin, G.G. Enzyme-coupled assays for simultaneous detection of nanomolar ATP, ADP, AMP, adenosine, inosine and pyrophosphate concentrations in extracellular fluids. Biochim. Biophys. Acta-Mol. Cell Res. 2012, 1823, 1967-1975. [CrossRef]

22. Karamohamed, S.; Nordstrom, T.; Nyren, P. Real-time bioluminometric method for detection of nucleoside diphosphate kinase activity. Biotechniques 1999, 26, 728-734. [CrossRef]

23. Hu, C.M.; Chang, Z.F. A bioluminescent method for measuring thymidylate kinase activity suitable for high-throughput screening of inhibitor. Anal. Biochem. 2010, 398, 269-271. [CrossRef] [PubMed]

24. Mohammadi, S.; Nikkhah, M.; Nazari, M.; Hosseinkhani, S. Design of a Coupled Bioluminescent Assay for a Recombinant Pyruvate Kinase from a Thermophilic Geobacillus. Photochem. Photobiol. 2011, 87, 1338-1345. [CrossRef] [PubMed]

25. Yamada, Y.; Goto, H.; Ogasawara, N. Adenosine kinase from human liver. BBA-Enzymol. 1981, 660, 36-43. [CrossRef]

26. Munch-Petersen, B.; Piskur, J.; Søndergaard, L. Four deoxynucleoside kinase activities from Drosophila melanogaster are contained within a single monomeric enzyme, a new multifunctional deoxynucleoside kinase. J. Biol. Chem. 1998, 273, 3926-3931. [CrossRef] [PubMed]

27. Matsuura, M.F.; Shaw, R.W.; Moses, J.D.; Kim, H.J.; Kim, M.J.; Kim, M.S.; Hoshika, S.; Karalkar, N.; Benner, S.A. Assays to Detect the Formation of Triphosphates of Unnatural Nucleotides: Application to Escherichia coli Nucleoside Diphosphate Kinase. ACS Synth. Biol. 2016, 5, 234-240. [CrossRef] [PubMed]

28. Leitão, J.M.M.; Esteves da Silva, J.C.G. Firefly luciferase inhibition. J. Photochem. Photobiol. B Biol. 2010, 101, 1-8. [CrossRef]

29. Sabini, E.; Ort, S.; Monnerjahn, C.; Konrad, M.; Lavie, A. Structure of human dCK suggests strategies to improve anticancer and antiviral therapy. Nat. Struct. Mol. Biol. 2003, 10, 513-519. [CrossRef] [PubMed]

30. Datta, N.S.; Shewach, D.S.; Hurley, M.C.; Mitchell, B.S.; Fox, I.H. Human T-lymphoblast deoxycytidine kinase: Purification and properties. Biochemistry 1989, 28, 114-123. [CrossRef] [PubMed]

31. Hazra, S.; Ort, S.; Konrad, M.; Lavie, A. Structural and kinetic characterization of human deoxycytidine kinase variants able to phosphorylate 5-substituted deoxycytidine and thymidine analogues. Biochemistry 2010, 49, 6784-6790. [CrossRef]

32. Iyidogan, P.; Lutz, S. Systematic Exploration of Active Site Mutations on Human Deoxycytidine Kinase Substrate Specificity. Biochemistry 2008, 47, 4711-4720. [CrossRef] [PubMed]

33. Mani, R.S.; Usova, E.V.; Eriksson, S.; Cass, C.E. Fluorescence studies of substrate binding to human recombinant deoxycytidine kinase. Nucleosides Nucleotides Nucleic Acids 2004, 23, 1343-1346. [CrossRef] [PubMed] 
34. Hellendahl, K.F.; Fehlau, M. Supplementary Information for "Semi-automated high-throughput substrate screening assay for nucleoside kinases". Zenodo 2021. Available online: https://doi.org/10.5281/zenodo.5363310 (accessed on 29 September 2021).

35. Serra, I.; Conti, S.; Piškur, J.; Clausen, A.R.; Munch-Petersen, B.; Terreni, M.; Ubiali, D. Immobilized Drosophila melanogaster Deoxyribonucleoside Kinase $(D m \mathrm{dNK})$ as a High Performing Biocatalyst for the Synthesis of Purine Arabinonucleotides. Adv. Synth. Catal. 2014, 356, 563-570. [CrossRef]

36. Munch-Petersen, B.; Knecht, W.; Lenz, C.; Søndergaard, L.; Piskur, J. Functional expression of a multisubstrate deoxyribonucleoside kinase from Drosophila melanogaster and its C-terminal deletion mutants. J. Biol. Chem. 2000, 275, 6673-6679. [CrossRef]

37. Eriksson, S.; Munch-Petersen, B.; Johansson, K.; Ecklund, H. Structure and function of cellular deoxyribonucleoside kinases. Cell. Mol. Life Sci. 2002, 59, 1327-1346. [CrossRef]

38. Kierdaszuk, B.; Krawiec, K.; Kazimierczuk, Z.; Jacobsson, U.; Johansson, N.G.; Munch-Petersen, B.; Eriksson, S.; Shugar, D. Substrate/Inhibitor Properties of Human Deoxycytidine Kinase (dCK) and Thymidine Kinases (Tk1 And Tk2) Towards the Sugar Moiety of Nucleosides, Including O'-Alkyl Analogues. Nucleosides Nucleotides 1999, 18, 1883-1903. [CrossRef]

39. Zhang, Y.; Secrist, J.A.; Ealick, S.E. The structure of human deoxycytidine kinase in complex with clofarabine reveals key interactions for prodrug activation. Acta Crystallogr. Sect. D Biol. Crystallogr. 2006, 62, 133-139. [CrossRef] [PubMed]

40. Eriksson, S.; Kierdaszuk, B.; Munch-Petersen, B.; Oberg, B.; Gunnar Johansson, N. Comparison of the substrate specificities of human thymidine kinase 1 and 2 and deoxycytidine kinase toward antiviral and cytostatic nucleoside analogs. Biochem. Biophys. Res. Commun. 1991, 176, 586-592. [CrossRef]

41. Nagai, S.; Takenaka, K.; Nachagari, D.; Rose, C.; Domoney, K.; Sun, D.; Sparreboom, A.; Schuetz, J.D. Deoxycytidine kinase modulates the impact of the ABC transporter ABCG2 on clofarabine cytotoxicity. Cancer Res. 2011, 71, 1781-1791. [CrossRef] [PubMed]

42. Knecht, W.; Mikkelsen, N.E.; Clausen, A.R.; Willer, M.; Eklund, H.; Gojković, Z.; Piškur, J. Drosophila melanogaster deoxyribonucleoside kinase activates gemcitabine. Biochem. Biophys. Res. Commun. 2009, 382, 430-433. [CrossRef] 